\title{
HOMOLOGY OF $\Omega^{n+1} \Sigma^{n+1} X$ AND $C_{n+1} X, n>0$
}

\section{BY FRED COHEN}

Introduction. Recently, Peter May, building on the work of Boardman and Vogt, has described the notion of a $\mathscr{C}_{n+1}$-space (see below) and has shown that $(n+1)$-fold loop spaces are $\mathscr{C}_{n+1}$-spaces [4]. This structure theory, together with my calculations of the equivariant homology of the spaces $C_{n+1}(p)[1]$, allows us to develop a complete theory of homology operations for $\mathscr{C}_{n+1}$-spaces and consequently for $(n+1)$-fold loop spaces. We construct functors, $W_{n}$ and $G W_{n}$, between appropriately defined categories (see \$2) which are left adjoints to the evident forgetful functors. ( $W_{n}$ and $G W_{n}$ are free functors.) By freeness, there are unique morphisms $\bar{\eta}_{*}$ and $\tilde{\eta}_{*}$ in our categories such that the following diagram is commutative, where $C_{n+1} X$ and $\alpha_{n+1}$ are as defined in $[4, \S \S 2,5]$.

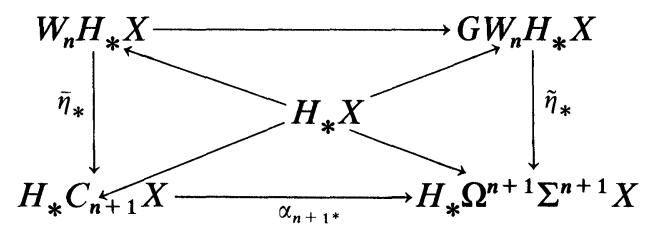

We show that $\bar{\eta}_{*}$ and $\tilde{\eta}_{*}$ are isomorphisms; consequently $W_{n}$ and $G W_{n}$ calculate $H_{*} C_{n+1} X$ and $H_{*} \Omega^{n+1} \Sigma^{n+1} X$ in closed form as functors of $H_{*} X, n>0$.

I wish to thank Peter May for his generous help. Most of the ideas and results presented here were conjectured by him some time ago; now his theory of operads allows fairly simple proofs of the conjectures.

1. Homology operations. All spaces are assumed to be compactly generated Hausdorff, with nondegenerate base point. All homology is taken with $Z_{p}$-coefficients for $p$ an arbitrary prime, unless otherwise stated. Modifications required when $p=2$ are stated in brackets.

Recall that a $\mathscr{C}_{n+1}$-space $(X, \theta)$ is a space $X$ together with an action $\theta$ of the little cubes operad $\mathscr{C}_{n+1}$ on $X ; \mathscr{C}_{n+1}$ denotes the category of $\mathscr{C}_{n+1}$ spaces. By definition, $X$ is equipped with $\Sigma_{j}$-equivariant maps

$$
\theta_{j}: \mathscr{C}_{n+1}(j) \times X^{j} \rightarrow X
$$

for all $j$, and $\theta_{2}(c): X^{2} \rightarrow X$ determines an $H$-space structure on $X$ for any

AMS (MOS) subject classifications (1970). Primary 55G99, 55D35. 
$c \in \mathscr{C}_{n+1}(2) . \mathscr{C}_{n+1}$ acts naturally on $(n+1)$-fold loop spaces [4]. In the following theorems we assume that all spaces are in $\mathscr{C}_{n+1}$.

THEOREM 1.1. There exist homomorphisms $Q^{s}: H_{q} X \rightarrow H_{q+2 s(p-1)} X$ $\left[H_{q} X \rightarrow H_{q+s} X\right], s \geqq 0$, for $2 s-q<n[s-q<n]$ which are natural with respect to maps of $\mathscr{C}_{n+1}$-spaces and satisfy the following properties:

(1) $Q^{s} x=0$ if $2 s<\operatorname{degree}(x)[s<\operatorname{degree}(x)], x \in H_{*} X$.

(2) $Q^{s} x=x^{p}$ if $2 s=\operatorname{degree}(x)[s=$ degree $(x)], x \in H_{*} X$.

(3) $Q^{s} \phi=0$ if $s>0$, where $\phi \in H_{0} X$ is the identity element.

(4) The external, internal, and diagonal Cartan formulas hold.

(5) The $Q^{s}$ are stable and the Kudo transgression theorem holds.

(6) The Adem relations hold.

(7) The Nishida relations hold.

Compare Theorem 1.1 with Theorem 1.1 of $\lceil 3\rceil$.

ReMARKs. When $X=\Omega^{n+1} Y$, the $Q^{s} x$ were defined, for $p=2$, by Araki and Kudo, and in the range $2 s-q \leqq n / p-1, x \in H_{q} X$, for $p>2$ by Dyer and Lashof. Milgram's calculations indicated that there were operations in the range $2 s-q \leqq n$. The "top" operation, for $2 s-q=n$ $[s-q=n]$, has exceptional properties and will be discussed below.

We note that Dyer and Lashof used the $(n+1)$-fold join of $\Sigma_{p}$, denoted $J^{n+1} \Sigma_{p}$, in their construction of the $Q^{s}$. However, any $\Sigma_{p}$-equivariant map $J^{n+1} \Sigma_{p} \rightarrow \mathscr{C}_{n+1}(p)$ is essential. Consequently, there is an obstruction to constructing all the $Q^{s}$ of Theorem 1.1 by use of $J^{n+1} \Sigma_{p}$. This obstruction arises from the presence of Browder operations $\lambda_{n}$, which are related by an obvious commutative diagram to the suspension of the Whitehead product.

THEOREM 1.2. There exist homomorphisms $\lambda_{n}: H_{q} X \otimes H_{r} X \rightarrow H_{q+r+n} X$ which are natural with respect to maps of $\mathscr{C}_{n+1}$-spaces and satisfy the foltowing properties:

(1) $\lambda_{0}(x, y)=x y-(-1)^{a r} y x$ for $x \in H_{q} X$ and $y \in H_{r} X$;

(2) $\lambda_{n}(x, y)=(-1)^{q r+1+n(q+r+1)} \lambda_{n}(y, x)$ for $x \in H_{q} X$ and $y \in H_{r} X$;

(3) $\lambda_{n}(\phi, x)=0=\lambda_{n}(x, \phi)$ where $\phi \in H_{0} X$ is the identity element and $x \in H_{*} X$.

(4) The analogues of the external, internal, and diagonal Cartan formulas hold:

where $|z|$ denotes the degree of $z$;

$$
\begin{aligned}
\lambda_{n}\left(x \otimes y, x^{\prime} \otimes y^{\prime}\right)= & (-1)^{\left|x^{\prime}\right|(|y|+n)} x x^{\prime} \otimes \lambda_{n}\left(y, y^{\prime}\right) \\
& +(-1)^{|y|\left(\left|x^{\prime}\right|+\left|y^{\prime}\right|+n\right)} \lambda_{n}\left(x, x^{\prime}\right) \otimes y y^{\prime},
\end{aligned}
$$

$$
\begin{aligned}
\lambda_{n}\left(x y, x^{\prime} y^{\prime}\right)= & (-1)^{\left|x^{\prime}\right|(|y|+n)}\left(x x^{\prime}\right)\left(\lambda_{n}\left(y, y^{\prime}\right)\right)+(-1)^{\left|x^{\prime}\right|\left|y^{\prime}\right|+|x||y|} y \lambda_{n}\left(x, y^{\prime}\right) x^{\prime} \\
& +(-1)^{|y|\left(\left|x^{\prime}\right|+\left|y^{\prime}\right|+n\right)}\left(\lambda_{n}\left(x, x^{\prime}\right)\right)\left(y y^{\prime}\right) \\
& +(-1)^{|x|\left(n+\left|x^{\prime}\right|+|y|\right)} \lambda_{n}\left(y, x^{\prime}\right) x y^{\prime}
\end{aligned}
$$


and

$$
\begin{aligned}
\psi \lambda_{n}(x, y)= & \sum(-1)^{n\left(|x|+\left|y^{\prime}\right|\right)+\left|x^{\prime \prime}\right|\left|y^{\prime}\right|} \lambda_{n}\left(x^{\prime}, y^{\prime}\right) \otimes x^{\prime \prime} y^{\prime \prime} \\
& +(-1)^{n\left|y^{\prime}\right|+\left|x^{\prime \prime}\right|\left|y^{\prime}\right|} x^{\prime \prime} y^{\prime \prime} \otimes \lambda_{n}\left(x^{\prime \prime}, y^{\prime \prime}\right)
\end{aligned}
$$

for $\psi x=\sum x^{\prime} \otimes x^{\prime \prime}$ and $\psi y=\sum y^{\prime} \otimes y^{\prime \prime}$.

(5) $\sigma_{*} \lambda_{n}(x, y)=\lambda_{n-1}\left(\sigma_{*} x, \sigma_{*} y\right)$ where $\sigma_{*}$ is the homology suspension.

(6) The Jacobi identity (which is the analog of the Adem relations) holds:

$$
\begin{aligned}
(-1)^{(q+n)(s+n)} \lambda_{n}\left[x, \lambda_{n}(y, z)\right]+(-1)^{(r+n)(q+n)} \lambda_{n}\left[y, \lambda_{n}(z, x)\right] & \\
& +(-1)^{(s+n)(r+n)} \lambda_{n}\left[z, \lambda_{n}(x, y)\right]=0
\end{aligned}
$$

for $x \in H_{q} X, y \in H_{r} X$, and $z \in H_{s} X ; \lambda_{n}\left[x, \lambda_{n}(x, x)\right]=0$ if $p=3$.

(7) The analogues of the Nishida relations hold:

and

$$
P_{*}^{s} \lambda_{n}(x, y)=\sum_{i+j=s} \lambda_{n}\left(P_{*}^{i} x, P_{*}^{j} y\right)
$$

$$
\beta \lambda_{n}(x, y)=\lambda_{n}(\beta x, y)+(-1)^{n+q} \lambda_{n}(x, \beta y) .
$$

(8) $\lambda_{n}\left[x, Q^{s} y\right]=0=\lambda_{n}\left[Q^{s} x, y\right]$ whenever $x, y \in H_{*} X$ and $Q^{s}$ is defined.

We next discuss the "top" operation $\xi_{n}$. The operation $\lambda_{n}$ is analogous to the bracket operation in a Lie algebra; $\xi_{n}$ is analogous to the restriction in a restricted Lie algebra.

THEOREM 1.3. There exists a function $\xi_{n}: H_{q} X \rightarrow H_{p q+n(p-1)} X$ defined when $n+q$ is even [ for all $q$ ] which is natural with respect to maps of $\mathscr{C}_{n+1}$-spaces and satisfies the following properties:

(1) If $X$ is a $\mathscr{C}_{n+2}$-space, then $\xi_{n} x=Q^{(n+q) / 2} x$, $\left[\xi_{n} x=Q^{n+q} x\right]$ for $x \in H_{q} X$.

(2) If we let $Q^{(n+q) / 2} x,\left[Q^{n+q} x\right]$, denote $\xi_{n} x, x \in H_{q} X$, then $\xi_{n}(x)$ satisfies formulas (1) through (6) of Theorem 1.1.

(3) Analogs (with error terms involving $\lambda_{n}$ ) of the Nishida relations hold.

(4) $\lambda_{n}\left[x, \xi_{n} y\right]=\operatorname{ad}_{n}^{p}(y)(x)$ where $\operatorname{ad}_{n}(y)(x)=\lambda_{n}(x, y)$ and inductively $\operatorname{ad}_{n}^{i}(y)(x)=\operatorname{ad}_{n}(y)\left(\operatorname{ad}_{n}^{i-1}(y)(x)\right)$ and $\lambda_{n}\left[x, \beta \xi_{n}(y)\right]=\lambda_{n}\left[x, \operatorname{ad}_{n}^{p-1}(y)(\beta y)\right]$.

(5) $\xi_{n}(x+y)=\xi_{n}(x)+\xi_{n}(y)+\sum_{1}^{p-1} d_{n}^{i}(x)(y)$ where

$$
\operatorname{id}_{n}^{i}(x)(y)=\sum \operatorname{ad}^{j_{1}}(x) \operatorname{ad}^{k_{1}}(y) \cdots \operatorname{ad}^{j_{r}}(x) \operatorname{ad}^{k_{r}}(y)(x) \quad \text { for } 1 \leqq i \leqq p-1
$$

with the sum taken over all sequences $\left(j_{1}, k_{1}, \ldots, j_{r}, k_{r}\right)$ such that $k_{l} \geqq 1$, $j_{1} \geqq 0$, and $j_{l} \geqq 1$ if $l>1$, and $\sum j_{l}=i-1, \sum k_{l}=p-i$. (Compare Jacobson's formula [5, p. 187] for restricted Lie algebras.)

(6) $\xi_{n}(k x)=k^{p} \xi_{n}(x)$ for $k \in Z_{p}$ whenever $\xi_{n} x$ is defined.

The Hopf algebras $H_{*} \Omega^{n+1} Y$ admit the conjugation $\chi=c_{*}$, where $c$ is the standard inverse map, and we have the following additional formulas. 
Proposition 1.4. On $H_{*} \Omega^{n+1} Y, Q^{s} \chi=\chi Q^{s}, \xi_{n} \chi=\chi \xi_{n}$, and $\chi \lambda_{n}(y, z)=$ $-\lambda_{n}(\chi y, \chi z)$.

2. Allowable $R_{n}$-structures. We describe some algebraic structures which are naturally suggested by the results above. We shall consider $Z_{p}$-modules and usually assume they are unstable $A$-modules, in the sense of homology, where $A$ is the Steenrod algebra.

Recall the definition of admissible monomials in the Dyer-Lashof algebra [3] and let $R_{n}(q)$ be the $Z_{p}$-subspace of $R$ having additive basis

$$
\left\{Q^{I} \mid I \text { admissible, } e(I) \geqq q, 2 s_{k}<n+q\left[s_{k}<n+q\right]\right\} .
$$

We do not give $R_{n}(q)$ any additional structure yet.

Definition 2.1. A module $L$ with Bocksteins is a $\beta$ restricted $\lambda_{n}$-algebra if $L$ is closed under a homomorphism $\lambda_{n}: L_{q} \otimes L_{r} \rightarrow L_{r+q+n}$ and a function $\xi_{n}: L_{q} \rightarrow L_{p q+n(p-1)}$, for $n+q$ even [all $\left.q\right]$ such that the Lie analogs (in the presence of Bocksteins), (2), (6), and (7) of Theorem 1.2, and the restriction analogues (in the presence of Bocksteins),(3)-(6) of Theorem 1.3 are satisfied. We note that there is no formula relating $\beta$ and $\xi_{n}$, but there is a Nishida relation for the identification of $\beta \lambda_{n}$. (If $p=2$, all reference to $\beta$ should be omitted.)

REMARKS. A $\beta$ restricted $\lambda_{n}$-algebra is an analogue of a restricted Lie algebra in the presence of Bocksteins.

DEFINITION 2.2. $M$ is an allowable $R_{n}$-module if there are homomorphisms

$$
Q^{s}: M_{q} \rightarrow M_{q+2 s(p-1)} \quad\left[M_{q} \rightarrow M_{q+s}\right]
$$

for $0 \leqq 2 s<q+n[s<q+n]$ such that $Q^{s}=0$ for $2 s<q[s<q]$ and the composition of the $Q^{s}$ satisfies the Adem relations. $M$ is an allowable $A R_{n}$-module if $M$ is an allowable $R_{n}$-module equipped with an $A$-action which satisfies the Nishida relations. $M$ is an allowable $A R_{n}$ algebra if $M$ is an allowable $A R_{n}$-module and a commutative algebra which satisfies the internal Cartan formula and (2) and (3) of Theorem 1.1. $M$ is an allowable $A R_{n} \Lambda_{n}^{\beta}$-Hopf algebra if $M$ is an unstable $A$-module which has the following properties:

(1) $M$ is a $\beta$ restricted $\lambda_{n}$-algebra which satisfies (2) and (3) of Theorem 1.2, and the Nishida analogues, (7) of Theorem 1.2 and (3) of Theorem 1.3.

(2) $M$ is an allowable $A R_{n}$-algebra which satisfies the internal Cartan formulas.

(3) $M$ is a cocommutative component coalgebra.

(4) The coproduct satisfies the diagonal Cartan formulas.

(5) $\lambda_{n}\left[Q^{s} x, y\right]=0=\lambda_{n}\left[x, Q^{s} y\right]$ for $x, y \in M$.

REMARK. Since the coproduct applied to $\lambda_{n}$ (Theorem 1.2(4)) requires the presence of products, we have chosen not to define separate notions 
of allowable $A R_{n} \Lambda_{n}^{\beta}$ coalgebras or algebras. Similar remarks apply to the definition of $W_{n} M$ below, due to the mixing of the $\xi_{n}$ and $Q^{s}$.

We require five free functors (adjoints to the forgetful functors) $L_{n}^{\beta}, D_{n}$, $V_{n}, W_{n}$, and $G$. The functors are defined on objects; the definitions on morphisms are evident.

(1) $L_{n}^{\beta}: Z_{p}$-modules with Bocksteins to $\beta$ restricted $\lambda_{n}$-algebras. Given $M$, set $L_{0}^{\beta} M$ equal to the subrestricted Lie algebra of $T(M)$ generated by $M$. $\left[T(M)\right.$ is the tensor algebra of $M$.] Suppose $L_{1}^{\beta} M$ is given. We inductively define $L_{n}^{\beta} M=s^{-1} L_{n-1}^{\beta} s M, n>1$, where $s M$ is a copy of $M$ with all elements raised one degree higher and $s^{-1} L_{n-1} s M$ is $L_{n-1} s M$ with all elements lowered one degree. Define

$$
\begin{aligned}
\lambda_{n}(x, y) & =s^{-1} \lambda_{n-1}(s x, s y), \quad \xi_{n}(x)=s^{-1} \xi_{n-1} s x, \\
\beta \xi_{n}(x) & =-s^{-1} \beta \xi_{n-1} s x, \quad \text { and } \quad \beta x=-s^{-1} \beta s x .
\end{aligned}
$$

If $n=1, p>2$, set $L_{1}^{\beta} M=s^{-1} L_{0}^{\beta} s M \oplus\left(\beta \xi_{1}\right)\left(s^{-1} L_{0}^{\beta} s M\right)$ as a $Z_{p}$-space, where $\left(\beta \xi_{1}\right)\left(s^{-1} L_{0}^{\beta} s M\right)$ has a basis consisting of elements $\left(\beta \xi_{1}\right)(x)$ of degree $2 p q-2$ for $x \in s^{-1} L_{0}^{\beta} s M$, degree $=2 q-1$. We make $L_{1}^{\beta} M$ into a $\beta$ restricted $\lambda_{1}$-algebra by requiring that $\lambda_{1}\left(y, \beta \xi_{1} x\right)=\left(y, \operatorname{ad}_{1}^{p-1}(x)(\beta x)\right)$. If $p=2$, all reference to $\beta \xi_{1}$ is ommitted and we set $L_{1} M=s^{-1} L_{0} s M$.

(2) $D_{n}: Z_{p}$-modules to allowable $R_{n}$-modules. Given $L$, set $D_{n} L=$ $\sum_{q>0} R_{n}(q) \otimes L_{q}$. The $R_{n}$-action is obvious.

(3) $V_{n}$ :Allowable $R_{n}$-modules to allowable $R_{n}$-algebras. Given $D$, define $V_{n} D$ as the free commutative algebra generated by $D$ modulo the two sides ideal generated by $\left\{x^{p}-Q^{s} x \mid 2 s=\operatorname{deg}(x)[s=\operatorname{deg}(x)]\right\}$.

We recall the definition of a cocommutative component coalgebra [3] and define $W_{n}$ in the following way:

(4) $W_{n}$ : Cocommutative component coalgebras over $A$ to allowable $A R_{n} \Lambda_{n}^{\beta}$-Hopf algebras: Given $M$, let $\eta$ denote the composite $Z_{p} \rightarrow M \rightarrow$ $L_{n}^{\beta} M \rightarrow D_{n}\left(L_{n}^{\beta} M\right)$. Define $W_{n} M$ as an allowable $A R_{n}$-algebra by $W_{n} M=$ $V_{n}\left(J D_{n} L_{n}^{\beta} M\right)$, where $J D_{n} L_{n}^{\beta} M=$ coker $\eta$. The additional structure is evident.

(5) $G$ : Allowable $A R_{n} \Lambda_{n}^{\beta}$-Hopf algebras to allowable $A R_{n} \Lambda_{n}^{\beta}$-Hopf algebras with conjugation. Given $W, G W$ is the localization of $W$ at $\pi_{0} W$.

3. $H_{*} \Omega^{n+1} \Sigma^{n+1} X$ and $H_{*} C_{n+1} X, n>0 . H_{*} \Omega^{n+1} \Sigma^{n+1} X$ is an allowable $A R_{n} \Lambda_{n}^{\beta}$-Hopf algebra with conjugation and $H_{*} C_{n+1} X$ is an allowable $A R_{n} \Lambda_{n}^{\beta}$-Hopf algebra. By freeness of $W_{n}$ and $G W_{n}$, there are unique morphisms $\bar{\eta}_{*}$ of allowable $A R_{n} \Lambda_{n}^{\beta}$-Hopf algebras and $\tilde{\eta}_{*}$ of allowable $A R_{n} \Lambda_{n}^{\beta}$-Hopf algebras with conjugation such that the diagram in the introduction is commutative.

THEOREM 3.1. For every space $X, \bar{\eta}_{*}: W_{n} H_{*} X \rightarrow H_{*} C_{n+1} X$ is an isomorphism of allowable $A R_{n} \Lambda_{n}^{\beta}$-Hopf algebras. 
THEOREM 3.2. For every space $X, \tilde{\eta}_{*}: G W_{n} H_{*} X \rightarrow H_{*} \Omega^{n+1} \Sigma^{n+1} X$ is an isomorphism of allowable $A R_{n} \Lambda_{n}^{\beta}$-Hopf algebras with conjugation.

COROLlARY 3.3. $\alpha_{n+1}: C_{n+1} X \rightarrow \Omega^{n+1} \Sigma^{n+1} X$ is a weak homotopy equivalence if $X$ is connected and a group completion in general.

The first part of the corollary was proven by May [4] and the second part was recently proven by Graeme Segal [unpublished].

The Bockstein spectral sequences of $C_{n+1} X$ and $\Omega^{n+1} \Sigma^{n+1} X$ have also been explicitly determined in terms of that of $X$.

Details of the above calculations (and more concrete descriptions of these homologies) will appear elsewhere.

\section{BIBLIOGRAPHY}

1. F. Cohen, Cohomology of braid spaces, Bull. Amer. Math. Soc. 79 (1973), 761-764.

2. N. Jacobson, Lie algebras, Interscience Tracts in Pure and Appl. Math., no. 10, Interscience, New York, 1962. MR 26 \#1345.

3. J. P. May, Homology operations on infinite loop spaces, Algebraic Topology, Proc. Sympos. Pure Math., vol. 23, Amer. Math. Soc., Providence, R.I., 1971, pp. 171-185.

4. - The geometry of iterated loop spaces, Lecture Notes in Math., vol. 271, Springer-Verlag, Berlin and New York, 1972.

Department of Mathematics, Northern Illinois University, DeKalb, Illinois 60115 